\title{
Repetitive sequences (RPSs) in the chromosomes of Candida albicans are sandwiched between two novel stretches, HOK and RB2, common to each chromosome
}

\author{
Ariya Chindamporn, $\uparrow$ Yoshiyuki Nakagawa, Ikuyo Mizuguchi, \\ Hiroji Chibana, $\neq$ Matsuko Doi $\$ and Kenji Tanaka
}

Author for correspondence: Yoshiyuki Nakagawa. Tel: +8152744 2460. Fax: +8152744 2459.
e-mail: yoshi@tsuru.med.nagoya-u.ac.jp

Laboratory of Medical Mycology, Research Institute for Disease Mechanism and Control, Nagoya University School of Medicine, Showa-ku, Nagoya 466, Japan

\begin{abstract}
A novel sequence designated HOK, which is next to the RPS, a repetitive sequence specific to Candida albicans, was cloned and sequenced. HOK hybridized with all of the chromosomes on which the RPSs were located, but did not hybridize with chromosome 3, which does not harbour any RPSs. Sequence determination revealed that a portion of HOK has significant homology with the B and $\mathrm{C} 1$ fragments of $\mathrm{Ca}$, which is used as a molecular epidemiological probe. A homology search of the deduced amino acids of HOK against the protein database showed partial homology with an isocitrate dehydrogenase of Saccharomyces cerevisiae, although an ORF large enough to encode the enzyme was not detected. To verify the existence of other sequences homologous with HOK, a portion of the HOK sequence was amplified using PCR. Sequence determination of the 41 clones from the PCR products resulted in at least six HOK-homologous clones. Another RPScontaining clone, RB2, was isolated from the Pstl-digested chromosome R or 1. It was determined that RB2a, one of the subclones from RB2, hybridized with all of the chromosomes, including chromosome 3, with which neither HOK nor RPS hybridized. The hybridization profile also showed that RPS is located between HOK and RB2a on chromosomes other than chromosome 3.
\end{abstract}

Keywords: repetitive sequence, RPS, physical map, Candida albicans

\section{INTRODUCTION}

A characteristic of the yeast Candida albicans is the variability in the size and number of its chromosomes (Magee \& Magee, 1987; Merz et al., 1988; Iwaguchi et al., 1990; Doi et al., 1992). This yeast has eight chromosomes per haploid, and lacks a sexual state in its life cycle, proliferating exclusively as a diploid cell (Scherer \& Magee, 1990; Iwaguchi et al., 1990; Chu et

†Present address: Department of Microbiology, Faculty of Medicine, Chulalongkorn University, Bangkok 10330, Thailand.

¥Present address: Department of Genetics and Cell Biology, University of Minnesota, St Paul, MN 55108, USA.

$\$$ Present address: School of Nursing, University of Shizuoka, Shizuoka 422, Japan.

The GenBank/EMBLDDBJ accession number for the $6 \mathrm{kbp}$ Smal fragment sequence reported in this paper is $D 63856$. al., 1993). We have analysed the genetic elements which might be involved in the variations in the chromosome size and in due course have found repetitive sequences, RPSs, which may serve as recombination hot spots generating their variability (Iwaguchi et al., 1992; Chibana et al., 1994; Chindamporn et al., 1995). The RPSs are located on all chromosomes except chromosome 3 (formerly 4 in our previous chromosome numbering system; Nakagawa, 1996) and are tandemarrayed in some chromosomes (Chibana et al., 1994). These sequences could have some functional significance, considering that they appear on almost all chromosomes. Many of the genetically identified centromeres are known to include repetitive sequences (Takahashi et al., 1992; Centola \& Carbon, 1994). A structural analogy between the RPS and human alpha satellite DNA, a possible human centromere, has been suggested (Chibana et al., 1994). However, Chu et al. (1993) suggested, based on SfiI macrorestriction frag- 


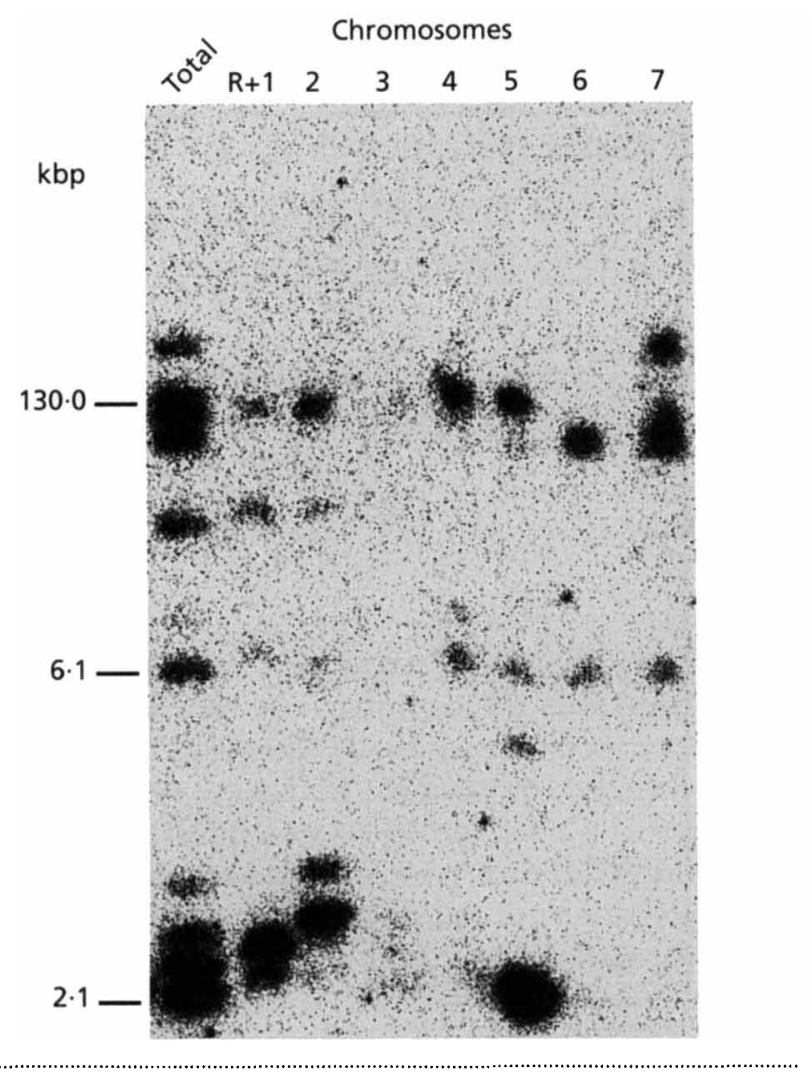

Fig. 1. Detection of the RPS-positive fragments in a Smaldigested chromosome. Each chromosome from FC18 fractionated by the first PFGE (see Methods) was digested with Smal. The digests were then fractionated by the second PFGE under conditions of $0.8 \%(\mathrm{w} / \mathrm{v})$ agarose and of 20-100 s with a ramped switching time for $15 \mathrm{~h}$ at $180 \mathrm{~V}$. After blotting to the membrane (Hybond-N; Amersham), Southern hybridization was performed using ${ }^{32}$ p-labelled RPS101 as a probe. Chromosome numbers are indicated on top and the numbers on the left represent the molecular sizes, which were estimated from the DNA standard size markers (see Methods). Chromosomes R and 1 were not separated under the PFGE conditions used here.

ment analysis, the possibility that the RPS or a closely linked locus was the site where chromosomal rearrangements occur.

While investigating RPSs and their boundary regions, we found that not only the RPSs but also the sequences neighbouring the RPS regions are also conserved among almost all chromosomes. Additionally, such sequences appeared to be conserved in all $C$. albicans strains examined so far. Here, we report the cloning and characterization of these newly discovered conserved fragments, which we call HOK and RB2. We also describe several HOK-related sequences which could be amplified from the genomic DNA of C. albicans. With the consideration that HOK, the RPSs and RB2 are contiguous on each chromosome, the functional significance of these structurally independent sequences is also discussed.

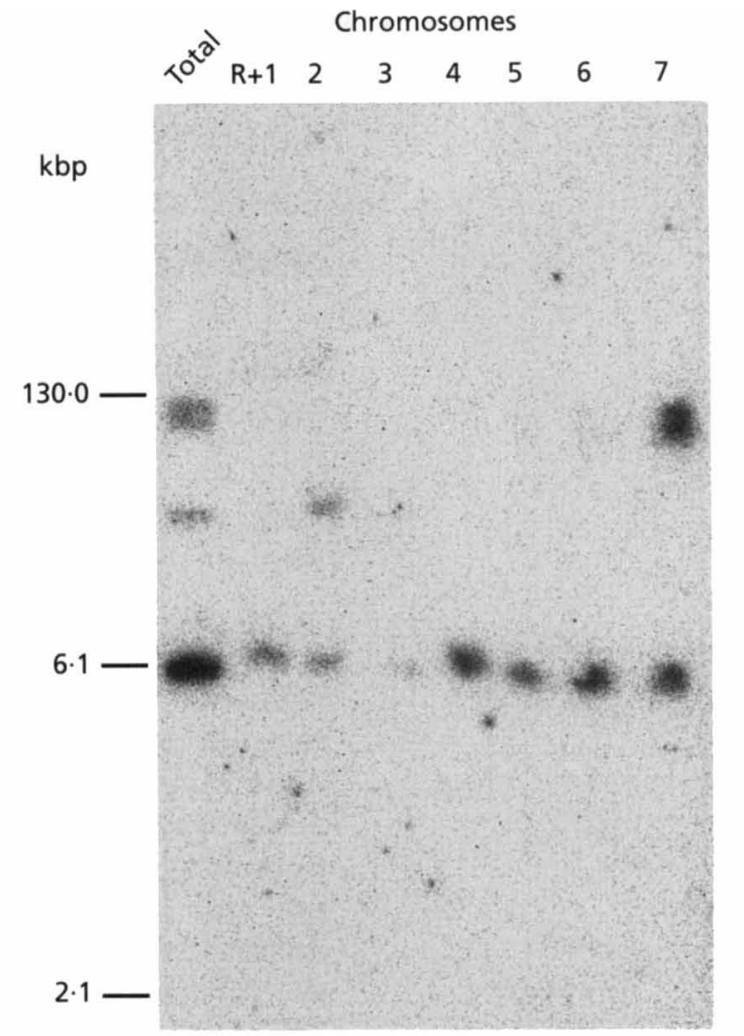

Fig. 2. Detection of the CS-positive fragments in a Smaldigested chromosome. The probe (RPS101) of the blot used in Fig. 1 was detached with a boiling solution of $0.5 \%$ SDS and $0.1 \times$ SSC twice. After confirmation that no signals were detected from the membrane, a ${ }^{32} \mathrm{P}$-labelled CS fragment was applied for Southern hybridization as a probe against the membrane. The numbers indicated are the same as those used in Fig. 1.

\section{METHODS}

Strains and culture conditions. C. albicans strains FC18 and 1006 and eight other clinical isolates of C. albicans (NUM46, $1000,1039,215,47,114,961$ and 812) were used (Iwaguchi et al., 1990). Cells from the late-exponential or early-stationary phase aerobically cultured in YPD $(1 \%$ yeast extract, $2 \%$ peptone, $2 \%$ dextrose) for $16 \mathrm{~h}$ at $30^{\circ} \mathrm{C}$ were used for DNA preparation.

Preparation of genomic DNA. The sample plug containing yeast chromosomal DNAs for PFGE was prepared by the method described previously (Iwaguchi et al., 1990). PFGE was carried out by the contour-clamped homogeneous electric field method using the Pulsaphor system with a hexagonal electrode array (Pharmacia-LKB Biotechnology) as described previously (Iwaguchi et al., 1990). Sample plugs were also used as a source of genomic DNA for restriction enzyme digestion. Chromosomes of Saccharomyces cerevisiae (X2180-1A), concatemeric DNA of lambda phage (Bio-Rad), and lambda phage DNA digested by EcoT14I (Takara Shuzo) were used as molecular size markers.

Smal digestion of individual chromosomes. Each chromosome of strain FC18 was separated by PFGE under conditions of $0.8 \%(\mathrm{w} / \mathrm{v})$ agarose and of $100 \mathrm{~s}$ at $170 \mathrm{~V}$ for 
(a)

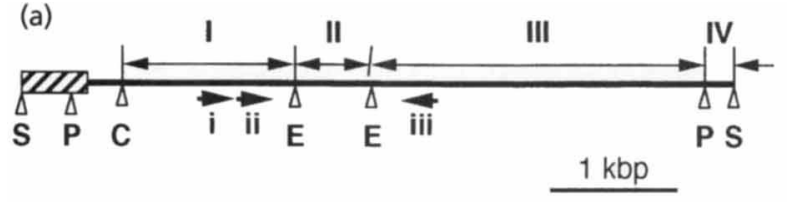

(b)
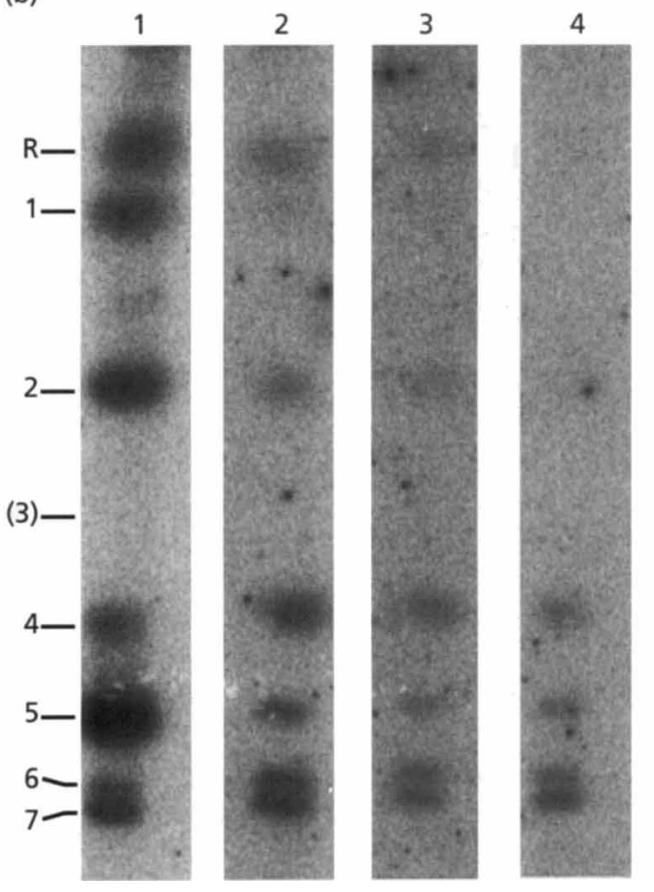

Fig. 3. Physical and genomic characterization of the $6 \mathrm{kbp}$ fragment. (a) Physical map of the $6 \mathrm{kbp}$ fragment. The hatched box indicates an RPS region. I, II, III and IV represent the CS subfragments. Subfragments I, II and III were used as probes in (b). i, ii and iii indicate primers 463,464 and 466 , respectively, used in Fig. 6. C, Clal; E, EcoRl; P, Pstl; S, Smal. (b) Hybridization profile of the CS subfragment on each chromosome. The chromosomes of FC18 were fractionated by PFGE (running conditions: $0.8 \%$ agarose, $300 \mathrm{~s}, 140 \mathrm{~V}, 40 \mathrm{~h}$, and $1200 \mathrm{~s}, 80 \mathrm{~V}$ $48 \mathrm{~h}$ ), which was followed by Southern hybridization using the following probes: 1 , RPS; 2 , fragment $I ; 3$, fragment $I 1 ; 4$, fragment III. The CS subfragments I, II and III are defined in (a).

$15 \mathrm{~h}, 300 \mathrm{~s}$ at $130 \mathrm{~V}$ for $24 \mathrm{~h}$ then $1000 \mathrm{~s}$ at $80 \mathrm{~V}$ for $48 \mathrm{~h}$. The running temperature was $12{ }^{\circ} \mathrm{C}$ throughout the experiment. The individual bands corresponding to each chromosome were cut out from the gel under UV light $(365 \mathrm{~nm})$ after staining the gel with ethidium bromide. The samples were subjected to Smal digestion as described previously (Chindamporn et al., 1995).

Southern hybridization. Southern hybridization was performed by using one of the following probes: a Pst I digest of pRPS101 for detection of RPSs (Chibana et al., 1994), a ClaI-SmaI fragment (CS fragment) of the $6 \mathrm{kbp}$ fragment which did not contain RPSs (for the $6 \mathrm{kbp}$ fragment and the CS fragment, see below and Fig. 3a), and RB2a, which was one of the subclones of the RB2 fragment (for RB2, see below). The probes were prepared by the random primer-labelling method with $\left[{ }^{32} \mathrm{P}\right] \mathrm{dCTP}$, and the hybridization signals were detected with an image analyser (BAS 2000; Fuji Photo Film).

Cloning of the $\mathbf{6}$ kbp fragment. Genomic DNA of FC18 in a sample plug was digested by $S m a \mathrm{I}$ as described above, and the

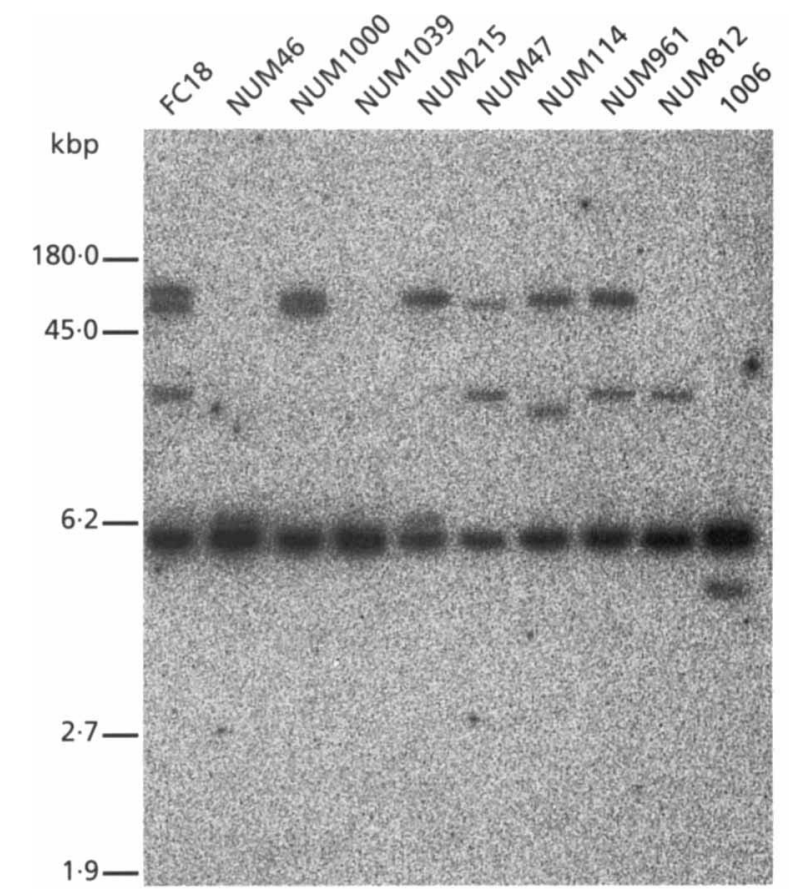

Fig. 4. Detection of the CS-homologous sequences in the clinical isolates of $C$. albicans. The genomic DNA from nine clinical isolates (NUM strains; Iwaguchi et al., 1990) and the standard strains 1006 and FC18 was digested with Smal and then separated by PFGE (see Methods). Blotting was performed followed by Southern hybridization using a ${ }^{32}$ p-labelled CS fragment. The numbers on the left indicate the estimated molecular sizes calculated from DNA standard size markers.

fragments were separated by PFGE. A band of approximately $6 \mathrm{kbp}$, as estimated by the molecular size marker, was cut out under UV light (365 nm). After phenol/chloroform extraction and ethanol precipitation, this fragment was integrated into the Smal site of pBluescript II SK(+) (Stratagene) and transformed into Escherichia coli XL-1 Blue (Stratagene). The white-coloured recombinants were selected on LB plates $(1 \%$ tryptone, $0.5 \%$ yeast extract, $1 \% \mathrm{NaCl}$ and $1.5 \%, \mathrm{w} / \mathrm{v}$, agar) containing $50 \mu \mathrm{g}$ ampicillin $\mathrm{ml}^{-1}, 20 \mu \mathrm{g} \mathrm{X}-\mathrm{Gal} \mathrm{m} \mathrm{m}^{-1}$ and $0.1 \mathrm{mM}$ IPTG. The recombinants were further screened by colony hybridization, using RPS as a probe, because the fragment in question was known to contain a part of the RPS (Chindamporn et al., 1995).

Cloning of the RB2 and RB3 fragments. The chromosome band containing $\mathrm{R}+1$ was excised from the gel after PFGE and digested with Pst $\mathrm{I}$. The resulting DNA was recovered by ethanol precipitation and integrated into a PstI-cleaved pUC18 vector. The RPS-positive clones were located by colony hybridization using ${ }^{32} \mathrm{P}$-labelled pRPS101 as a probe. Of these, a clone which contained a larger insert size of plasmid than RPS was screened further. By using a similar procedure, the RB3 fragment was isolated from chromosome 2.

Sequencing. Subclones of the $6 \mathrm{kbp}$ fragment were constructed by either excision of the $6 \mathrm{kbp}$ fragment with appropriate kinds of restriction enzymes or sequential deletion with exonuclease III and mung bean nuclease (Takara Shuzo). The sequences of all the clones were determined with a DNA Sequencer (model 373A; ABI) using Taq Dye Primer Cycle 

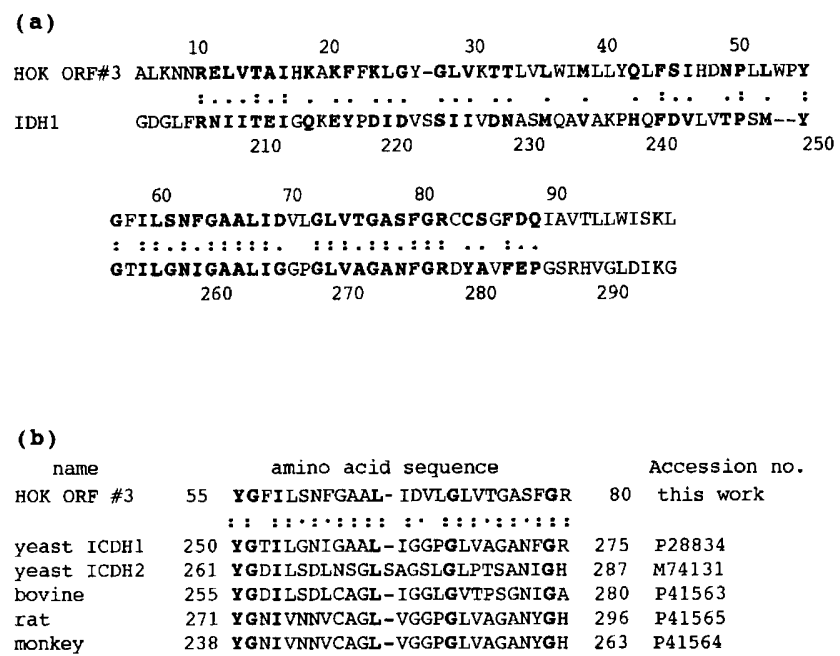

Fig. 5. Homology search of HOK against the protein database. (a) Homology of the deduced amino acid sequence from nucleotide positions 2253-2546 of HOK with the amino acid sequence of the isocitrate dehydrogenase of 5 . cerevisiae (IDH1; Cupp \& McAlister-Henn, 1992). The number above the HOK sequence is the amino acid position of a hypothetical ORF of HOK, and the number below the sequence of IDH1 is the amino acid position of Idh1p. Identical amino acids are shown by colons and physico-chemically related amino acids are shown by stops. Intervals (-) were introduced for maximum matching between both sequences. (b) Alignment of a portion of the HOK sequence with amino acid sequences of the isocitrate dehydrogenase of several organisms. Identical amino acids among the six sequences are indicated by bold letters. The numbers on both sides of the sequences indicate the position of the amino acid in each protein.

Sequencing kits (Perkin Elmer). Analyses of the data were carried out with the software GENETYX-MAC (Software Development).

Amplification and cloning of the HOK-related sequences. The HOK-related sequences were amplified by PCR. The primer sets used were 5'-GAGACCATGTCATATTGCCCCC-3' (463; forward, position 1870-1891 in the $6 \mathrm{kbp}$ fragment), 5'-CATTTCGGGAGCCATATTAGTCGC-3' (464; forward, 1934-1957) and 5'-CACACCCCCAGTTTGATATCCAG-3' (466; reverse, 2134-2156). PCR was performed using Taq polymerase and its accompanying buffer (Takara Shuzo) with the Thermal Cycler (model PJ-2000; Perkin Elmer). Amplification conditions were as follows: $94{ }^{\circ} \mathrm{C}$ for $5 \mathrm{~min}$ prior to the cycling step; 35 cycles of $94{ }^{\circ} \mathrm{C}$ for $20 \mathrm{~s}, 50^{\circ} \mathrm{C}$ for $20 \mathrm{~s}$ and $72^{\circ} \mathrm{C}$ for $90 \mathrm{~s}$ for amplification; followed by $37^{\circ} \mathrm{C}$ for $5 \mathrm{~min}$ for extension. After amplification, the PCR products were isolated by agarose gel electrophoresis and GeneClean II (Bio101), and digested with EcoRI. The digested products were then introduced into EcoRI-cleaved and alkaline-phosphatase-treated pBluescript II $\mathrm{SK}(+)$ and transformed into XL-1 Blue.

\section{RESULTS}

\section{Detection and cloning of a new conserved sequence}

It has been reported that the RPS on each chromosome is located in a limited region, and that on chromosome 5 (chromosome 6 in our previous numbering system) it is flanked by $6.1 \mathrm{kbp}$ and $130 \mathrm{kbp}$ SmaI fragments
(Chindamporn et al., 1995). To obtain further information about the RPS-flanking regions on the other chromosomes, each chromosome previously fractionated by PFGE was treated with SmaI. The resulting fragments were separated by a second PFGE, followed by Southern hybridization using RPS as a probe (Fig. 1). The bands were categorized into two groups: one was derived from the RPSs themselves, ranging in size from $1.9 \mathrm{kbp}$ to $2.7 \mathrm{kbp}$ (Chibana et al., 1994), and the other was thought to be derived from the boundary regions of the RPS $(s)$ on the respective chromosomes. As shown in Fig. 1, bands of approximately $6 \mathrm{kbp}$ in size were detected in almost all chromosomes, except for chromosome 3 , indicating that fragments similar in size to the SmaI fragments are present on almost all chromosomes. As these fragments also contain a part of an RPS sequence, it could be interpreted that the SmaI recognition site is at a locus which is the same distance from the RPS region on each chromosome. If this is the case, it may be expected that a portion of the sequence in those SmaI fragments other than just the RPS region is also conserved, because it is unlikely that it is only by accident that the $S m a I$ site is present at the same distance from the RPS region on each chromosome. To verify this assumption, we attempted to clone a new SmaI fragment of approximately $6 \mathrm{kbp}$ that could be hybridized with an RPS probe from the genomic DNA of strain FC18.

\section{CS, a conserved fragment}

The $6 \mathrm{kbp}$ SmaI cloned fragment included a portion of the RPS. Therefore, by digestion of the fragment with ClaI, we constructed a fragment which did not contain a portion of an RPS. This new fragment, designated CS, was then used as a probe for detection of other members of conserved sequences in the genome. After dehybridization of the RPS probe shown in Fig. 1, the same blot was hybridized with a CS probe. As shown in Fig. 2 , at the position that corresponds to a size of approximately $6 \mathrm{kbp}$, distinct signals were detected in chromosomes $\mathrm{R}+1,2,4,5,6$ and 7 . A few extra bands at about $15 \mathrm{kbp}$ and 60 and $80 \mathrm{kbp}$ were detected in chromosomes 2 and 7, respectively. The positions of the signals of about $6 \mathrm{kbp}$ in size which were detected by the CS probe coincided with those detected by the RPS probe. These data indicate that the novel sequence which is distinct from but is neighbouring the RPS is conserved in most of the chromosomes. Physical mapping of the $6 \mathrm{kbp}$ fragment showed that the Pst I recognition sites are near the $S m a I$ sites at both ends of the fragment. A similar result was obtained by CS probe hybridization against PstI-digested chromosomes (data not shown). This indicates that both SmaI and Pst I recognition sites are conserved on almost all chromosomes. Because the CS fragment is quite large, approximately $5.4 \mathrm{kbp}$, we asked which portion(s) of the CS is(are) responsible for the hybridization profile. To answer this question, a CS fragment was divided into four portions: fragment I, $1.5 \mathrm{kbp}$ ClaI-EcoRI; fragment II, $0.6 \mathrm{kbp}$ EcoRI-EcoRI; fragment III, $3 \mathrm{kbp}$ 
EcoRI-PstI; and fragment IV, $0 \cdot 2 \mathrm{kbp} P$ stI-SmaI (shown in Fig. 3a). Each fragment except IV was used as a probe to hybridize the separated chromosomes of FC18. The results are shown in Fig. 3(b). All three probes hybridized to the FC18 chromosomes 4, 5, 6 and 7 with a similar hybridization pattern, suggesting that almost the whole region of the CS fragment is conserved. It was suggested that the $6 \mathrm{kbp}$ fragment cloned here was derived from one of four chromosomes (chromosome 4, 5,6 or 7), because the intensity of the signal from chromosomes R, 1 and 2 was relatively weak.

\section{The CS-homologous sequence is conserved among different $C$. albicans strains}

To examine whether or not the sequence of the CS fragment was also conserved in other strains, the following experiment was performed. Chromosomal DNA from clinical isolates obtained at the University Hospital and from the standard strain 1006 was digested with SmaI, followed by Southern hybridization using the CS fragment as a probe. As shown in Fig. 4, two to four bands were detected in all strains used. The band corresponding to the $6 \mathrm{kbp}$ fragment was clearly observed with high intensity in all strains examined. We also observed that the CS fragment could hybridize with the C. albicans-related species Candida stellatoidea (data not shown). In addition to the $6 \mathrm{kbp}$ fragment, most of the strains have one to three extra bands corresponding to the CS fragment hybridization. In strain FC18, these signals are derived from the chromosomes $R+1$ and 7 (see Fig. 2).

\section{Sequencing analysis and a new conserved sequence, HOK}

We determined the base sequence of the $6 \mathrm{kbp} \mathrm{SmaI}$ fragment. The fragment is $5782 \mathrm{bp}$ and the $\mathrm{G}+\mathrm{C}$ content is $35 \mathrm{~mol} \%$. One boundary of the $6 \mathrm{kbp}$ fragment included $523 \mathrm{bp}$ of the RPS, and we were able to determine the boundary of the RPS. The sequence of an RPS portion in the $6 \mathrm{kbp}$ fragment did not match with any of the other RPSs already sequenced and with alt, the inner short repetitive sequences in RPS. Thus, we named the $5259 \mathrm{bp}$ fragment, the sequence in which the RPS portion was excluded from the $6 \mathrm{kbp}$ fragment, as HOK. A homology search using the FASTA program revealed a high degree of homology with the $\mathrm{B}$ fragment of $\mathrm{Ca} 3$, which has frequently been used as a molecular epidemiological probe (Sadhu et al., 1991; Anderson $e t$ al., 1993). Most of the B fragment was included in HOK. Recently, Lockhart et al. (1995) sequenced the C1 portion of the $\mathrm{C}$ fragment, another EcoRI fragment of $\mathrm{Ca} 3$, and reported that $\mathrm{C} 1$ contains an RPS element. By comparing the sequence of $\mathrm{C} 1$ with that of the $6 \mathrm{kbp}$ fragment, we confirmed that the $\mathrm{C} 1$ fragment contains portions of both the RPS and HOK, with a total homology of $95 \%$. Interestingly, the homology between the HOK-related portion of the $\mathrm{C} 1$ fragment and HOK was $79 \%$ (97 bp in $123 \mathrm{bp}$ ), while that between the RPS- related portion of the $\mathrm{C} 1$ and the $6 \mathrm{kbp}$ fragment was $99 \%$ (851 bp in $859 \mathrm{bp}$ ). An ORF search showed three small ORFs within HOK. Surprisingly, the polypeptide sequence determined from one of the ORFs analysed by the FASTA program showed significant homology with part of the isocitrate dehydrogenase protein of the yeast S. cerevisiae, as shown in Fig. 5(a) (Cupp \& McAlisterHenn, 1992).

\section{Multiplicity of HOK-related sequences}

A CS hybridization experiment (Fig. 2) showed that HOK-related sequences were located on many chromosomes. If there is a multiplicity of HOK-related sequences in the C. albicans genome, it would be possible to obtain different clones when PCR is performed using part of the HOK sequence for amplification. We amplified 1.3 or $1.4 \mathrm{kbp}$ fragments containing the region flanked by EcoRI using primer sets $463-466$ and $464-466$ (see Fig. 3a; data not shown). After treatment of the PCR products with EcoRI, they were integrated into a pBluescript II SK(+) vector. The 233 clones isolated were classified into 41 groups according to the size of the insert, the digestion profile with HindIII and Bam HI, and a Southern blot analysis using the CS fragment as a probe. The sequences of all 41 clones were determined, and they could be categorized into seven kinds of sequence, including the original sequence of HOK (Fig. 6). These results strongly suggest that there are at least seven kinds of HOK-related sequences in the $C$. albicans genome. Homologies among these sequences ranged from $82 \%$ to $97 \%$ (data not shown). Of the seven groups, two groups (646620 and 636602) had a $23 \mathrm{bp}$ insertion, and another two clones (646608 and 646611) had an additional EcoRI recognition site.

\section{Another kind of conserved sequence, RB2}

From comparing Fig. 1 with Fig. 2, we expected that most signals larger than $6 \mathrm{kbp}$ would be derived from the other boundary of RPS, opposite to HOK. Therefore, we tried to isolate these fragments under conditions which would allow them to hybridize with the RPS probe but not the CS probe and be larger than an RPS (see Methods). As a result, we obtained two clones, named RB2 and RB3. The first of these clones, RB2, was digested using EcoRI and was subcloned into a pBluescript II $S K(+)$ vector. One of the subcloned fragments, designated RB2a and about $4 \mathrm{kbp}$ in size, hybridized with all chromosomes of strain FC18, including chromosome 3 which was not labelled by the RPS or by HOK, as shown in Fig. 7. Considering that the RB2a fragment does not hybridize with $\mathrm{HOK}$, this result strongly suggests that in all chromosomes except chromosome 3, structurally independent, conserved sequences are consecutively arranged in tandem. A partial sequence analysis of the RB2 fragment has shown that the orientation of the sequence of the RPS within RB2 was inverted compared to that within HOK. This result also strongly suggests that RB2 is located on the opposite side of the RPSs to HOK (data not shown). 


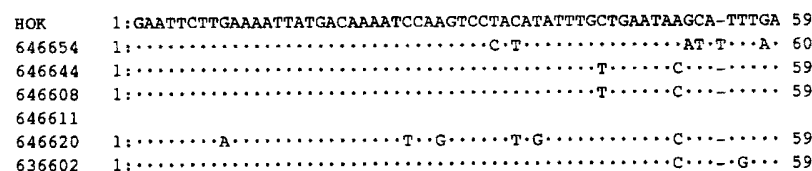

BOK 60: TTTTGCTT-TGAAGAC-AACAGAGAATTGGTTACTGCTAT-CCACAAAGCTAAATTTTT 116

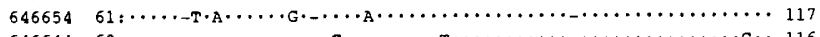

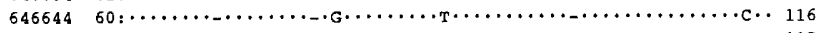

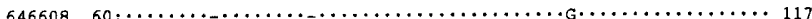

646611

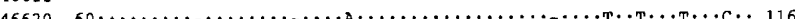

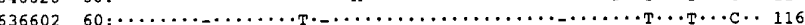

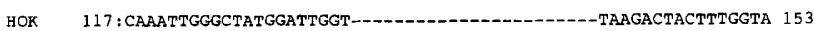

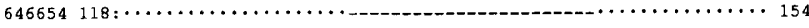

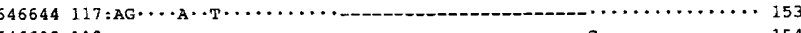
$646608118: \cdots \cdots \ldots \ldots \ldots \ldots \ldots \ldots$ 646611

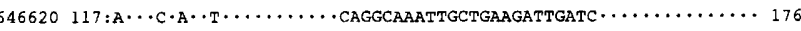

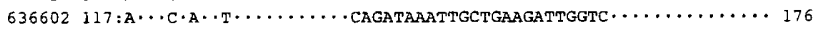

HOK 154:CTGTGGATAATGCTTTTATACCAGCTGTTCTCAATCCATGACAATCCATTGTTGTGGCCG 213

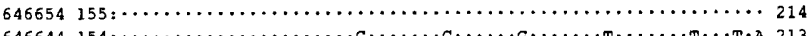

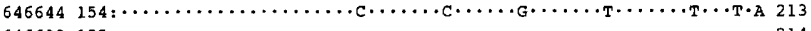

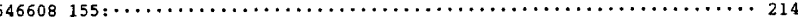
646611

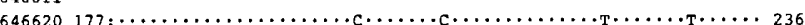

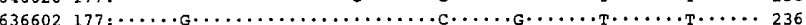

HOK 214:TATGGTTHTATCCTATCCAACTTTGGTGCTGCATTAATAGATGTCTTG-GGPTTGTTAC 271

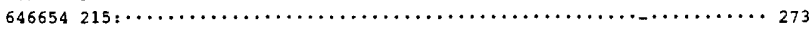

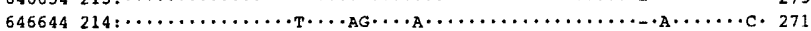

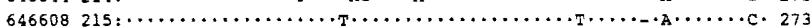
646611

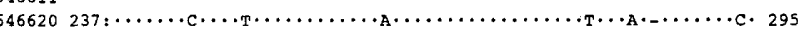

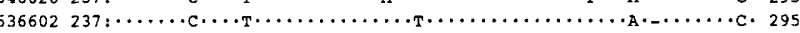

HOK 272 : AGGTGCTAGC-TTTGGTAGATGCTGTTCTGGT-TTTGACCAGATTGCCGTCACTTTACTT 330

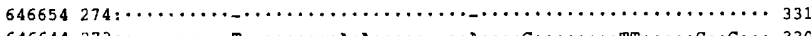
$646644273: \cdots \cdots \cdots \cdot+\cdots \cdots \cdots \cdot$.

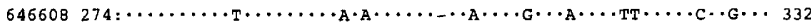
646611

$646620296: \cdots \cdots \cdots \cdots+\mathrm{T} \cdots \cdots \cdots \cdots \cdot \mathrm{AC} \cdots \cdots \cdot \mathrm{A}_{\mathrm{A}} \cdots \cdots \cdots \cdot \mathrm{G} \cdots \cdots+\mathrm{TT} \cdots \cdots \cdot \mathrm{C} \cdot \mathrm{G} \cdots 354$

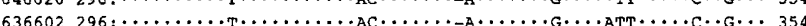

צOK 331:TGGATATCAAAGCTAAAAATTCAGAATTGACTTAATGGTAATT-CC-CATTTTATGATTC 388

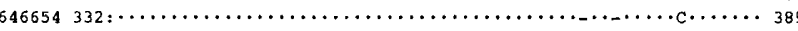

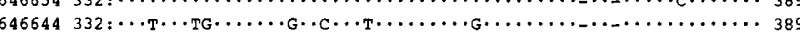

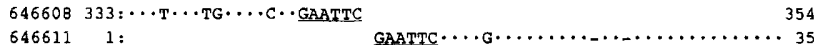

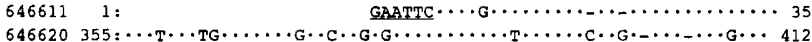

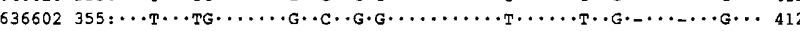

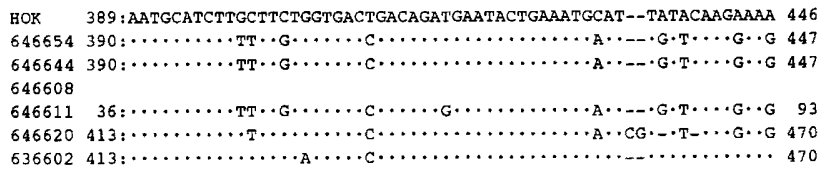

HOK 447 : TTTGAATTAGTTGGTT-CTAATGCAATCT-TATGTGTTTCTGAACGGGATATGTTGTGGA 504

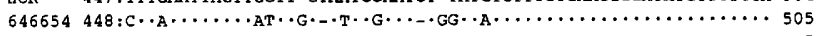

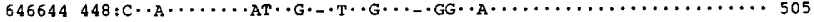
646608

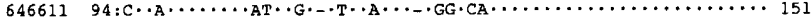

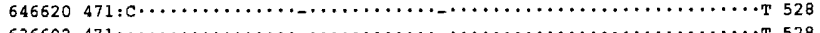

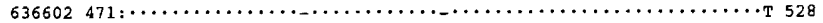

HOR 505 : CAATATCAAAGAGTGGTT-TTPTTAAACT-GGATCAG-GATCCAATCTTTCC-TAGTCAC 560

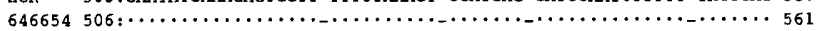

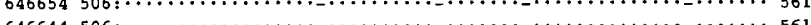

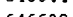

646600

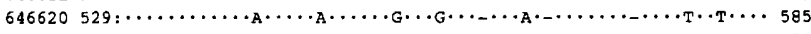

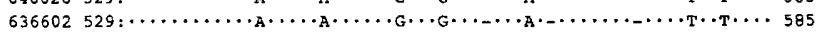

HOK 561:CTAG-CTATAACAAAAGCAAGTAGTTGAGGTCATTGCTACTTTCGCATGGTTGATGTTT 619

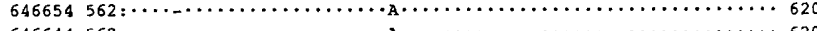

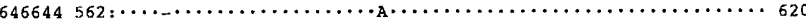
646608

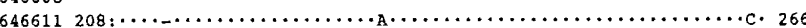

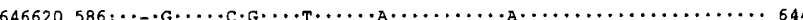

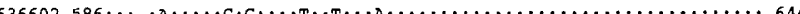

HOK 620:TAGGTAATAAGAATTC 635 (12)

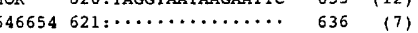

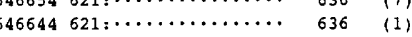

646608

$646611267: \cdots \ldots \ldots \ldots \ldots \ldots, 282$ (1)

$646620645: \cdots \cdots \cdots \cdots \cdots \cdots \cdots, 660 \quad(3)$

$636502645, \ldots \ldots \ldots \ldots \ldots \ldots, 660$

\section{DISCUSSION}

Our studies are summarized in Fig. 8(a), which shows a schematic drawing of the three sequences, HOK, RPS and RB2a, in each chromosome. We have identified new conserved sequences, $\mathrm{HOK}$ and $\mathrm{RB} 2$, neighbouring the RPS and have determined the base sequence of HOK. RPSs are one of the repetitive sequences in C. albicans which are located on most of the chromosomes. The number of RPS units on each chromosome is estimated from previous results, although an accurate count of the RPS units on chromosome 5 has not been made (Iwaguchi et al., 1992; Chibana et al., 1994; Chindamporn et al., 1995). RPSs are clustered in some chromosomes, but it is still not known whether or not the other new sequences (HOK and RB2a) are located at a single locus. Currently, in the laboratory of P. T. Magee (University of Minnesota, MN, USA), a detailed physical map is being established using a fosmid library. According to the map information, at least on chro- mosome 7, HOK or its related stretch is located on two loci (P. T. Magee, H. Chibana \& S. Scherer, personal communication). This is in accordance with our findings that two bands are observed in chromosome 7 upon hybridization using a CS fragment as a probe (see Fig. 2). Similarly to the case of the RPS, we could not detect a signal from chromosome 3 against the CS fragment hybridization. However, we have shown that the RB2a fragment can hybridize with all chromosomes, including chromosome 3. As shown in Fig. 7, the RB2a hybridization signals from chromosome 3 are relatively weak. Therefore we have concluded that only a portion of the RB2a sequence is homologous in chromosome 3 , whereas the overall sequence seems to be homologous with RB2a in other chromosomes. The boundary region between the RPSs and RB2a has not been characterized.

Conservation of the SmaI site on the opposite side of the RPS on each HOK led us to assume that a conserved region would extend beyond the SmaI site. Considering 
(a)

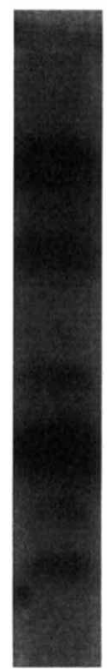

(b)

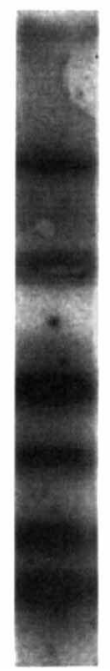

(c)

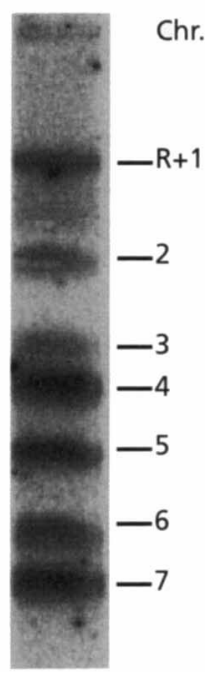

Fig. 7. Comparison of the hybridization profiles among three conserved sequences. The chromosomes of FC18 were fractionated by PFGE followed by blotting to the nylon membrane. Hybridization was performed using ${ }^{32}$ p-labelled probes. (a) RPS101; (b) CS fragment; (c) RB2a fragment. Note that chromosome 3 was labelled with RB2a.

the possible function of HOK (and the other conserved sequences), not only HOK but the region which extends over HOK might also be essential for some proper function.

When a CS fragment is hybridized with the SmaIdigested genomic DNA of several clinical strains, we noticed that a few signals specific to the strain appear in addition to the $6 \mathrm{kbp}$ band common to every strain. Therefore, CS labelling patterns could be used for the delineation of clinical isolates of C. albicans.
The Ca3 molecular epidemiological probe developed by D. R. Soll's group (Sadhu et al., 1991) contains both the RPS- and the HOK-related sequences. We have confirmed that the hybridization patterns of the RPS and of HOK are different from each other (Figs 1 and 2). Therefore, it may be possible to distinguish RPS-positive signals from HOK-positive ones among the $\mathrm{Ca} 3$-positive signals. Fig. 8(b) represents the relationship between HOK, RPS and Ca3. Approximately one-half of the $6 \mathrm{kbp}$ fragment (from bp 2831 to 5782 ) had homology $(93 \%)$ with the B fragment of $\mathrm{Ca} 3$, and another portion (from bp 1 to 645 ) was homologous with the $\mathrm{C} 1$ fragment of Ca3. Lockhart et al. (1995) showed the C1 fragment of $\mathrm{Ca} 3$ as a Sacl-cleaved subfragment of the C fragment of Ca3. According to the physical map of the $\mathrm{C}$ fragment, the $\mathrm{C} 2$ fragment is expected to be homologous with a part of HOK. The EcoRI recognition site of the C2 fragment, for which the sequence has not been reported, would then coincide with that of position 2202 of HOK, according to our sequence data. The homology of the $6 \mathrm{kbp}$ fragment with portions of the $\mathrm{C} 1 \mathrm{fragment}$ was $79 \%$ in the HOK region and $99 \%$ in the RPS region. This low degree of homology for HOK might be due to the different origin of the strains: $\mathrm{Ca} 3$ was derived from 3153a (Sadhu et al., 1991) and our $6 \mathrm{kbp}$ fragment was from $\mathrm{FC18}$, which was a clinical isolate from the cervix (Whelan \& Magee, 1981). In contrast, the high degree of homology for the RPS between the two independent strains suggests that this sequence may be instrumental in some cellular function(s). We have not yet examined whether HOK or $\mathrm{RB} 2 \mathrm{a}$ is homologous with another molecular epidemiological probe, 27A (Scherer \& Stevens, 1988); however, it has been shown that many fosmids which can hybridize with the RPS are also labelled with HOK or RB2a (S. Scherer, H. Chibana \& P. T. Magee, personal communication).

Sequence analysis of the $6 \mathrm{kbp}$ fragment revealed three short ORFs; one was significantly homologous to a

(a)

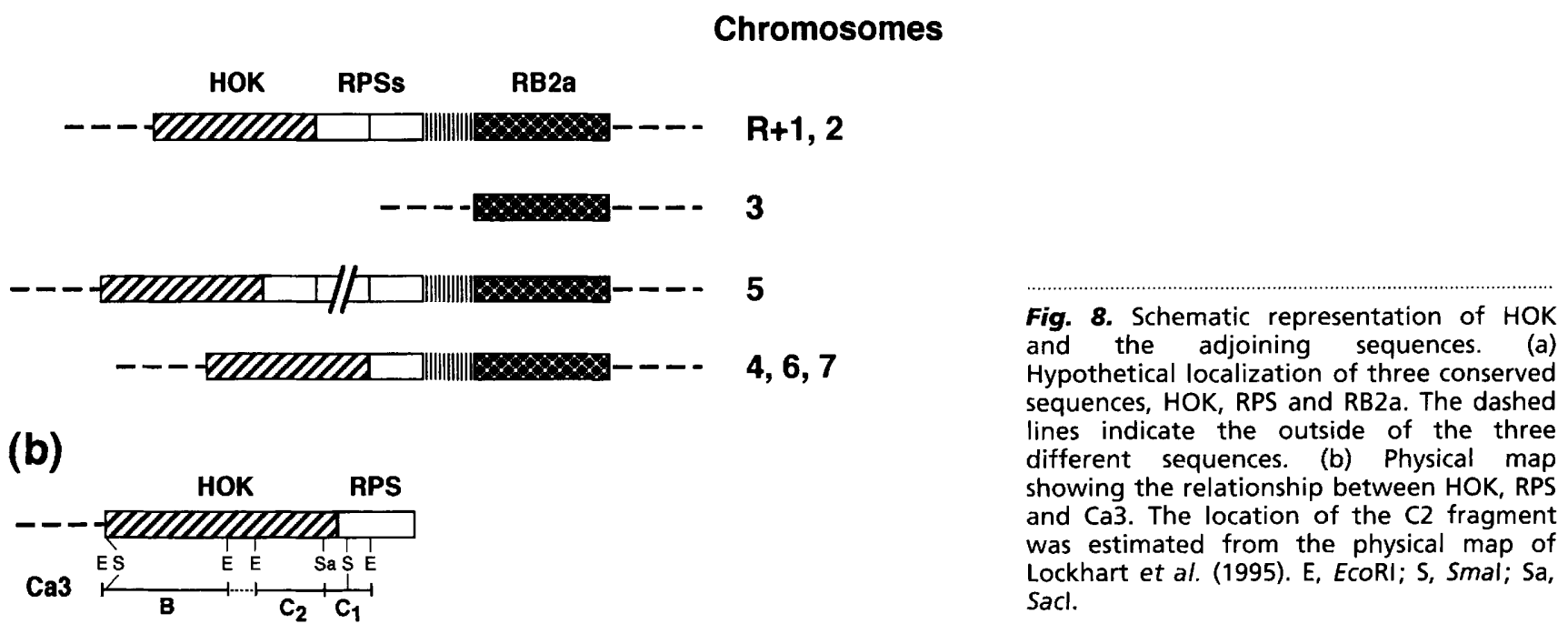


portion of the isocitrate dehydrogenase gene of $S$. cerevisiae. The conserved region shown in Fig. $5(\mathrm{~b})$ contains the amino acid sequences responsible for binding isocitrate as a substrate as well as the magnesium-ion- and $\mathrm{NAD}^{+}$-binding sites necessary for enzyme reactions (Hurley et al., 1991). Nevertheless, we do not think that this region of HOK functions as an active enzyme for several reasons. First, this ORF is too short to encode a full-size enzyme. It also contains many stop codons but no ATG initiation codon. Finally, it is not likely that this kind of gene is located on many chromosomes. Elzinga et al. (1993) reported that isocitrate dehydrogenase is an RNA-binding protein, although it is not known which region is involved in the actual binding to the RNA.

Another feature of the HOK sequence is the existence of the successive AT-stretch from positions 1098 to 1202 (AAA TAATTTTTGC CATACAAAAT TATTTTTCTT AGTAAATTTT TTAGTACTTT TCTAGCGCTT AGATTTTTGC TAAATCTATT TTCTTTTACA ACTATTTTCT TA) (80\% AT content). CDEII, one of the components of the centromere of the yeast S. cerevisiae, is highly AT-rich ( $>90 \%$; Clarke, 1990). However, no homologous region corresponding to CDE-I or CDE-III, the centromeric components in $S$. cerevisiae, has been found near the AT-rich region of HOK. Recently, Candida glabrata, one of the pathogenic yeasts, has been shown to harbour a Saccharomyces-like centromere (Kitada et al., 1996).

It is known that several successive tracts of adenine nucleotide cause a curvature in the secondary structure of a DNA string, resulting in retardation of migration in a gel matrix such as the agarose in gel electrophoresis (Hagerman, 1990). This may be reflected in our experience of estimating the size of the $6 \mathrm{kbp}$ fragment as approximately $6.1 \mathrm{kbp}$, based on the migration profile in agarose gel electrophoresis, but finding that the actual size by sequence determination is approximately $5.8 \mathrm{kbp}$.

A curved DNA is also known to be one of the characteristics of kinetochore in Schizosaccharomyces pombe (Ueki et al., 1993). Therefore, we tested the possibility that HOK may be one component of the centromere. However, we could not obtain any plasmids containing the RPS and/or HOK which were stable in the C. albicans host. The finding that another sequence, $\mathrm{RB2a}$, which is not related to the RPS or to HOK, is conserved among all the chromosomes of strain FC18, along with its boundary region, suggests that RB2a could be a newly discovered component of the machinery for cellular or chromosomal function together with HOK and RPS. Recently, it has been proposed that the name MRS (major repeat sequence) should be given to three successive conserved regions (P. T. Magee, personal communication). To elucidate the cellular and chromosomal function of the MRS, a detailed analysis of the RB2a homologous sequences in the other chromosomes will be necessary.

\section{REFERENCES}

Anderson, J., Srikantha, T., Morrow, B., Miyasaki, S. H., White, T. C., Agabian, N., Schmid, J. \& Soll, D. R. (1993). Characterization and partial nucleotide sequence of the DNA fingerprinting probe Ca3 of Candida albicans. J Clin Microbiol 31, 1472-1480.

Centola, M. \& Carbon, J. (1994). Cloning and characterization of centromeric DNA from Neurospora crassa. Mol Cell Biol 14, 1510-1519.

Chibana, H., Iwaguchi, S.-I., Homma, M., Chindamporn, A., Nakagawa, Y. \& Tanaka, K. (1994). Diversity of tandemly repetitive sequences due to short periodic repetitions in the chromosomes of Candida albicans. J Bacteriol 176, 3851-3858.

Chindamporn, A., Nakagawa, Y., Homma, M., Chibana, H., Doi, M. \& Tanaka, K. (1995). Analysis of the chromosomal localization of the repetitive sequences (RPSs) in Candida albicans. Microbiology 141, 469-476.

Chu, W.-S., Magee, B. B. \& Magee, P. T. (1993). Construction of an Sfi macrorestriction map of the Candida albicans genome. $J$ Bacteriol 175, 6637-6651.

Clarke, L. (1990). Centromeres of budding and fission yeasts. Trends Genet 6, 150-154.

Cupp, J. R. \& McAlister-Henn, L. (1992). Cloning and characterization of the gene encoding the IDH1 subunit of $\mathrm{NAD}^{+}$. dependent isocitrate dehydrogenase from Saccharomyces cerevisiae. J Biol Chem 267, 16417-16423.

Doi, M., Homma, M., Chindamporn, A. \& Tanaka, K. (1992). Estimation of chromosome number and size by pulsed-field gel electrophoresis (PFGE) in medically important Candida species. $J$ Gen Microbiol 138, 2243-2251.

Elzinga, S. D. J., Bednarz, A. L., van Oosterum, K., Dekker, P. J. T. \& Grivell, L. A. (1993). Yeast mitochondrial $\mathrm{NAD}^{+}$-dependent isocitrate dehydrogenase is an RNA-binding protein. Nucleic Acids Res 21, 5328-5331.

Hagerman, P. J. (1990). Sequence-directed curvature of DNA. Annu Rev Biochem 59, 775-781.

Hurley, J. H., Dean, A. M., Koshland, D. E., Jr \& Stroud, R. M. (1991). Catalytic mechanism of $\mathrm{NADP}^{+}$-dependent isocitrate dehydrogenase: implications from the structures of magnesiumisocitrate and $\mathrm{NADP}^{+}$complexes. Biochemistry 30, 8671-8678.

Iwaguchi, S.-I., Homma, M. \& Tanaka, K. (1990). Variation in the electrophoretic karyotype analysed by the assignment of DNA probes in Candida albicans. J Gen Microbiol 136, 2433-2442.

Iwaguchi, S.-I., Homma, M., Chibana, H. \& Tanaka, K. (1992). Isolation and characterization of a repeated sequence (RPS1) of Candida albicans. J Gen Microbiol 138, 1893-1900.

Kitada, K., Yamaguchi, E. \& Arisawa, M. (1996). Isolation of a Candida glabrata centromere and its use in construction of plasmid vectors. Gene 175, 105-108.

Lockhart, S. R., Fritch, J. J., Meier, A. S., Schroppel, K., Srikantha, T., Galask, R. \& Soll, D. R. (1995). Colonizing populations of Candida albicans are clonal in origin but undergo microevolution through $\mathrm{C} 1$ fragment reorganization as demonstrated by DNA fingerprinting and C1 sequencing. J Clin Microbiol 33, 1501-1509.

Magee, B. B. \& Magee, P. T. (1987). Electrophoretic karyotypes and chromosome numbers in Candida species. J Gen Microbiol 133, 425-430.

Merz, W. G., Connelly, C. \& Hieter, P. (1988). Variation of electrophoretic karyotypes among clinical isolates of Candida albicans. J Clin Microbiol 26, 842-845.

Nakagawa, Y. (1996). Chromosome structure in Candida 
albicans. In Genes and Genomes in Medically Important Fungi, pp. 25-30. Edited by K. Tanaka, H. Yamaguchi \& P. T. Magee. Tokyo: Foundation for the Advancement of International Science.

Sadhu, C., McEachern, M. J., Rustchenko-Bulgac, E. P., Schmid, J., Soll, D. R. \& Hicks, J. B. (1991). Telomeric and dispersed repeat sequences in Candida yeasts and their use in strain identification. $J$ Bacteriol 173, 842-850.

Scherer, S. \& Magee, P. T. (1990). Genetics of Candida albicans. Microbiol Rev 54, 226-241.

Scherer, S. \& Stevens, D. (1988). A Candida albicans dispersed, repeated gene family and its epidemiologic applications. Proc Natl Acad Sci USA 85, 1452-1456.
Takahashi, K., Murakami, S., Chikashige, Y., Funabiki, H., Niwa, O. \& Yanagida, M. (1992). A low copy number central sequence with strict symmetry and unusual chromatin structure in fission yeast centromere. Mol Biol Cell 3, 819-835.

Ueki, N., Momoi, H., Yamada, H. \& Mizuno, T. (1993). Distribution of bent DNA structures in the fission yeast centromere. Gene 132, 247-250.

Whelan, W. L. \& Magee, P. T. (1981). Natural heterozygosity in Candida albicans. J Bacteriol 145, 896-903.

Received 19 August 1997; revised 12 November 1997; accepted 19 November 1997. 